\title{
Los límites de la globalización capitalista: la necesidad de cambiar la relación entre la sociedad y la naturaleza
}

\author{
Melissa Salgado \\ Departamento de Economía \\ UCA, San Salvador
}

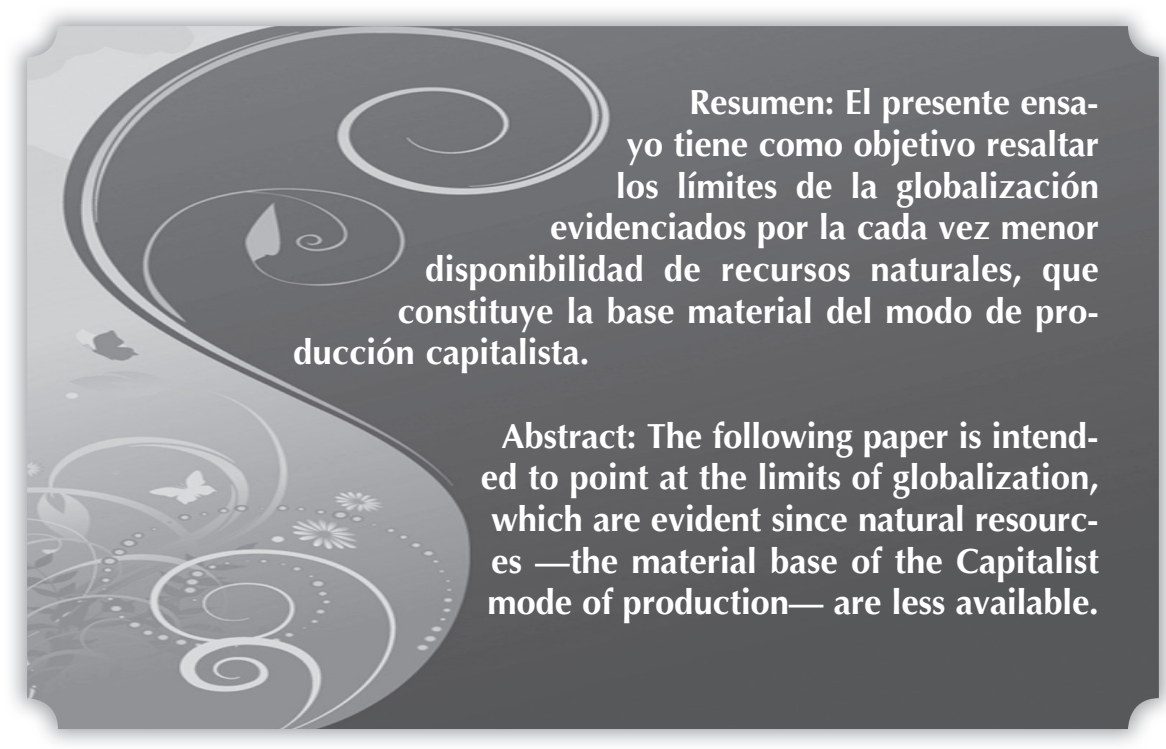

\section{Introducción}

El presente ensayo tiene como objetivo resaltar los límites de la globalización evidenciados por la cada vez menor disponibilidad de recursos naturales, que constituye la base material del modo de producción capitalista. Para ello se destacarán cuatro ejes fundamentales para determinar la necesidad del cambio de relación entre la sociedad y la naturaleza, la energía como la base energética del sistema, la producción y su falta de consideración de la naturaleza como base material, el crecimiento económico sin límites y la distribución desigual de los recursos naturales entre los países.

El trabajo se dividirá en tres partes. La primera de ellas es un marco teórico en el cual se contex- 
tualizarán los procesos involucrados en el modo de producción capitalista que determinan los límites de las economías capitalistas. En la segun- da parte se fundamenta la necesidad del cambio de la relación entre la sociedad y la naturaleza, para desembocar en las conclusiones.

\section{Marco teórico}

La globalización económica capitalista tiene límites constituidos por aspectos económicos, políticos, sociales, culturales y medioambientales, ya que la globalización tiene lugar en un solo espacio que es el planeta. Ante una superficie finita, con recursos naturales finitos, con un ecosistema que depende de ciclos de energía con caducidad, los procesos económicos de producción, consumo y distribución están limitados.

Como señala el autor Elmar Altvater: "las formas de vida y de producción de la moderna sociedad industrial no son compatibles con la naturaleza" (Altvater 2002: 308), ya que la globalización en el planeta es un proceso destructivo del medio ambiente.

La globalización económica ha requerido de desregulación de políticas ambientales, para que el modo de producción no vea obstaculizado su proceso de acumulación. En este sentido, las economías capitalistas se desarrollan bajo la contradicción entre el proceso de acumulación y el proceso de destrucción de la base material.

Para evidenciar la necesidad de un cambio en la relación de la so- ciedad con la naturaleza, se hará referencia a cuatro ejes fundamentales del modo de producción capitalista: la energía, la producción, el crecimiento económico y la distribución.

Energía

Desde la primera revolución industrial, el sistema capitalista extrae su energía de los combustibles fósiles que "poseen una ventaja decisiva para la dinámica de la acumulación [...] la cantidad de energía puede ser concentrada de manera comparativamente sencilla" (Altvater 2002: 310). Esto es clave para la realización de la lógica del desarrollo capitalista, al someter la base energética a procesos de rentabilidad.

Sin embargo, existen desventajas de establecer el sistema de producción en combustibles fósiles. Un primer inconveniente consiste en que son extraídos de una reserva finita y agotable. En segundo lugar, se trata de una fuente de energía que, una vez utilizada, no tiene un proceso reversible, hecho que sugiere la aplicación de la segunda ley de la termodinámica: la entropía. Ante esta situación, Altvater señala que "no pueden cumplirse las duras 
condiciones de la termodinámica de la sustentabilidad, es decir, una balanza de entropía equilibrada en la utilización de combustibles fósiles" (Altvater 2002: 311).

La revolución industrial se sustenta en los combustibles fósiles, dada su ventaja de almacenamiento y acumulación. Pero a su vez, esta revolución industrial ve su base energética inmersa en la supresión: "la revolución industrial, encuentra su dimensión y sus barreras en las reservas finitas de recursos y las reducciones del planeta tierra" (Altvater 2002: 316).

Por lo tanto, se resalta lo cuestionable de un orden social que se base en recursos energéticos fósiles, no durables, agotables, irreversibles, dicho orden social se condena a sí mismo a la destrucción de su propia base material, y con ello a la condiciones materiales que garantizan la vida humana.

El sistema energético basado en los combustibles fósiles es la plataforma de la producción capitalista y ésta se encuentra en crisis.

\section{Producción}

La producción capitalista ha generado un incremento del consumo productivo de recursos naturales, es decir, un mayor ritmo de extracción y transformación de materia, de gasto de energía y de producción de desechos, que, en buena medida, se explica por "la no valorización de los recursos naturales ha impedido que los desequilibrios ecológicos, la pérdida de fertilidad de los suelos, la destrucción de los recursos no renovables, etc. repercutan directamente en el valor del capital y en la formación de precios; al mismo tiempo la acumulación y revalorización del capital se ha ido reflejando en la degradación del patrimonio de recursos naturales" (Leff 2003: 347).

Con ello se enfatiza que los recursos naturales, al no representar un costo de producción por no ser productos del valor, es decir, del trabajo humano, son objeto de depredación sin ningún miramiento sobre su existencia en el futuro.

La destrucción ecológica, la sobreexplotación de los recursos naturales y la degradación ambiental han sido efecto del proceso de acumulación de capital, que se explica principalmente por dos aspectos: "por una parte, la no valorización de la naturaleza ha favorecido la sobreexplotación de los recursos [...] por otra parte, las crisis de realización del capital y la innovación tecnológica llevan a reducir los ciclos de recomposición y revalorización del capital, lo cual ha generado procesos de producción destructiva que multiplican y aceleran los procesos de extracción de recursos no renovables" (Leff 2003: 351). En resumen, tanto la falta de valorización de la naturaleza como la disminución del tiempo de los procesos productivos 
son los grandes causantes de la destrucción del medio ambiente.

Los procesos productivos de diferenciación y la división del trabajo cada vez más profundizada, hacen que el régimen de tiempo no solo se caracterice por la aceleración sino también por la fragmentación: "mucho se tiene que hacer al mismo tiempo y lo mismo se debe hacer en muchos tiempos" (Altvater 2002: 317). Esto agudiza el problema ecológico, ya que la flexibilidad del tiempo requiere de mayor cantidad de energía y recursos naturales.

En las economías capitalistas, el tiempo y el espacio carecen casi totalmente de importancia como barreras naturales: "la abstracción del tiempo y el espacio es la característica de la realidad concreta de la economía de mercado capitalista globalizada" (Altvater 2002: 321).

Por otra parte, la producción del sistema capitalista también es un ejemplo de la aplicabilidad de la segunda ley de la termodinámica, la entropía, ya que se trata de un proceso de utilización de energía y de recursos naturales de forma irreversible, una vez producidos, no vuelven a su estado natural. Además, la disminución de los tiempos en dichos procesos, incrementa el grado de entropía.

La disminución del tiempo, vista como sinónimo de aceleración en el proceso productivo, se vuelve necesaria a consecuencia de la competencia de los mercados globales: "ya no existe el futuro, éste es presentizado, quedando sólo como valor presente descontado, es decir, desfuturizado" (Altvater 2002: 380). El peligro de ello es que no se consideran las futuras generaciones.

El análisis de la naturaleza como límite para la producción retoma la cuestión de los límites al crecimiento, "cada vez más la crisis ambiental adquiere un carácter global —desequilibrios ecológicos, crisis alimentaria, avance de la pobreza, miseria extrema, etc." (Leff 2003: 348).

\section{Crecimiento económico}

Se entiende por crecimiento económico el ritmo de volumen de producción y consumo que tenga una población en un determinado periodo. Pero ambos procesos se encuentran dentro de los límites de la sustentabilidad de los ecosistemas del planeta.

Por sustentabilidad se entiende una forma "de realizar un registro cualitativo $y$, sobre todo, cuantitativo de las reservas de energía y materia y de su distribución" (Altvater 2002: 322). Dicho concepto no considera la capacidad de regeneración de los ecosistemas en una dimensión temporal. En realidad, este nuevo sistema de reglas se rige por la directriz de la sustentabilidad, que trata de adaptar los ritmos de los ciclos naturales a la valorización económica y a su reproducción. 
La industrialización ha producido una variedad de valores uso que posibilita un nivel de satisfacción de necesidades única en la historia de la humanidad, pero el sistema económico y social se expande contra los limites del medio ambiente, "el resultado del crecimiento económico se ha obtenido a un costo más elevado que él mismo, el precio de la prosperidad es demasiado alto, la prosperidad resulta demasiado cara" (Altvater 2002: 326). En otras palabras, existe una contradicción entre crecimiento económico y conservación de los recursos naturales.

\section{Distribución}

Se debe destacar la diferencia entre los problemas ambientales de los países ricos y los pobres, y también la distribución desigual de los costos ambientales "entre naciones, regiones y clases sociales, generados por el intercambio desigual entre las mercancías tecnológicas del norte y las naturales del sur, induciendo la sobreexplotación de los recursos" (Leff 2003: 353).

El mercado produce inequidad de ingresos que genera derechos desiguales a participar en la naturaleza. "La desigualdad del consumo de la naturaleza se puede medir con indicadores, del presupuesto energético total de que dispone la humanidad, un centroeuropeo utiliza, por cabeza, aproximadamente 6 kilovatios; un norteamericano, 11 kilovatios; un chino, 0.8 y un africano, 0.08 kilovatios. Si se realizara una distribución equitativa, cada ciudadano dispondría de 1.5 kilovatios" (Altvater 2002: 325).

Es imposible que haya una distribución equitativa del consumo de los recursos naturales y energía en el mismo nivel de los países industrializados. Es una ilusión creer que los países en desarrollo llegarán a obtener los mismos estándares de vida que los países desarrollados: "el medio ambiente está agotado, el consumo del medio ambiente debe reducirse, la industrialización no puede tener acceso a todas las regiones y naciones del planeta [...] ponerse al corriente de la industrialización es la esperanza de todas las sociedades en vías de desarrollo, es un proyecto irrealizable" (Altvater 2002: 326). Ello responde a que la competitividad a la que se aspira en los mercados globales exige capacidad tecnológica, altos estándares de calificación, gestión eficiente. Las exigencias de la vida moderna se basan en una variedad de consumo que sólo puede ser producida de forma industrial. Por lo tanto, el desarrollo equivale a una industrialización generalizada: "la industrialización y la modernización son un privilegio oligárquico del que algunas sociedades pueden gozar y otras no" (Altvater 2002: 329). La industrialización es para una pequeña parte de la humanidad, en contra del resto de la misma.

El sistema de acceso a los recursos a nivel internacional respon- 
de a que "sólo quien dispone de dinero puede tener acceso a la escasa oferta de recursos [...] quienes no dispongan de dinero quedan fuera en su mayoría" (Altvater 2002: 336). En este sentido, no se puede esperar del mecanismo del mercado una distribución equitativa, ni económica ni ecológicamente.

El sistema capitalista global ha acentuado las desigualdades a escala planetaria, entre el norte desarrollado y el sur poco desarrollado, entre las economías de extracción y las economías de producción industrial, entre los propietarios del dinero y los deudores, entre los ricos y los pobres.

El siglo XX ha sido catalogado como el siglo del crecimiento, en el que "las tasas de crecimiento en la segunda mitad del siglo no solo fueron tan altas como nunca antes en la milenaria historia de la humanidad [...] sino que la lógica de la acción individual en la producción y consumo, así como la lógica de las instituciones en el mercado, la política y el espacio social, son totalmente determinadas por el crecimiento positivo de la dinámica de acumulación del capital" (Altvater 2002: 384). Empero, el crecimiento económico se ha encargado de demostrar que no es la respuesta a los problemas de la distribución desigual de los recursos, Al contrario, dicho proceso aumenta la concentración de la riqueza y de la pobreza. "Los limites del crecimiento no están dados por las fuerzas de la naturaleza sino que son elementos de un proceso social" (Altvater 2002: 385), es decir de la relación de la sociedad con la naturaleza.

\section{Relación entre la sociedad y la naturaleza}

Para Marx, "el trabajo es [...] un proceso entre el hombre y la naturaleza, un proceso en que el hombre media, regula y controla su metabolismo con la naturaleza" (Marx, 2005:215). Los objetos del trabajo son la naturaleza y las materias primas y el medio de trabajo es un conjunto de instrumentos que el hombre interpone entre él y el objeto de trabajo y le sirve para efectuar su actividad. De acuerdo con Marx, el proceso de trabajo tiene como fin producir valores de uso, en donde el humano se apropia de lo natural para satisfacer sus necesidades $y$ esta relación hombre naturaleza es una condición eterna y natural.

Marx explica así cómo el proceso de producción se convierte en proceso de formación de valor. El capitalista se apodera de la fuerza de trabajo del obrero y al ser ésta su propiedad en el momento del proceso de producción, le permite valorizar el trabajo, pagando un salario que no corresponde al valor del trabajo expresado en el proceso productivo, haciéndolo trabajar 
jornadas de trabajo más extensas. Es decir, Marx observa al hombre como objeto productor de valor mediante su fuerza de trabajo aplicada sobre la naturaleza, a la cual transforma y da valor.

El trabajo ejerce así una función de mediador entre la sociedad y la naturaleza, y de esta manera "la destrucción de la naturaleza es un efecto sobredeterminado por la explotación del trabajo" (Leff 2003: 336). En este sentido, el modo de producción capitalista deja de forma oculta la cuestión ambiental, ya que no permite relacionar la destrucción de la naturaleza como la destrucción de la base material de todo proceso económico y productivo.

Dentro del concepto del trabajo socialmente necesario, la medida de valor construye una relación de explotación entre capital y trabajo, pero esta medida de valor excluye las condiciones de producción de la naturaleza. "La teoría marxista de la producción no incorpora los procesos naturales y culturales a la producción del valor, por ello es incapaz de valorar el patrimonio de los recursos naturales y culturales como condiciones de sustentabilidad para la reproducción ampliada" (Leff 2003: 338). Para Marx, la oferta natural de recursos carecía de valor y de precio, al no incorporar el trabajo humano como única fuente y sustancia de valor.

Marx anticipó los efectos del modo de producción capitalista en la destrucción de la base de los recursos naturales del planeta y en la pérdida de fertilidad de los suelos, a través de la teoría de la renta diferencial -en donde el suelo más estéril determina el precio de la renta en el mercado-, aunque no planteó un desarrollo teórico de esta limitante del modo de producción capitalista, quedando invisible con ello la destrucción de la base de recursos y del equilibrio ecológico, soporte de todo proceso económico y productivo.

Se debe de reestablecer una nueva relación entre la sociedad y la naturaleza en donde se incorpore ésta última como "sustancia de valor de los procesos naturales y determinante de las fuerzas productivas" (Leff 2003: 344).

\section{Conclusiones}

Las economías capitalistas se desarrollan bajo la contradicción entre el proceso de acumulación y el proceso de destrucción de la base material.
La energía es un recurso finito e irreversible, hecho que sugiere la aplicación de la segunda ley de la termodinámica: la entropía. En la actualidad, "los límites de la capa- 
cidad de carga de las reservas para las emisiones gaseosas, líquidas y sólidas del proceso de producción y de consumo se han acotado dramáticamente" (Altvater 2002: 308).

En el proceso productivo capitalista también es aplicable la entropía, ya que dichos procesos requieren de recursos y energías que, una vez utilizados, no regresan a su estado original. Asimismo, la desvaloración de la naturaleza y la mayor velocidad de los procesos productivos son los grandes causantes de la depredación despiadada del medio ambiente.

El crecimiento económico, bajo el modo de producción capitalista, trata de adaptar los ritmos de los ciclos naturales a la valorización económica y a su reproducción, por lo que existe una contradicción entre el crecimiento económico y la preservación del medio ambiente.

El mecanismo de asignación del mercado depende de la disponibilidad de ingresos de las naciones. En este sentido, no se puede esperar una distribución equitativa ni económica ni ecológicamente, "los países pobres no podrán alcanzar los niveles de consumo de los países ricos imitando sus patrones no sustentables de producción y consumo de recursos" (Leff 2003: 349). Asimismo, las condiciones de dependencia económica que sufren los países pobres, agravadas por el endeudamiento externo, generan mayores presiones sobre ritmos de extracción de recursos sin límites.

El modo de producción capitalista relaciona la sociedad y la naturaleza a través del trabajo, que para Marx es la única fuente de valor, pero esta determinación excluye la reproducción de la naturaleza como base material del sistema, es decir los recursos naturales y la energía quedan invisibilizados en el sistema capitalista, por no contar con una valoración cuantitativa. Por ello, es necesario un cambio en la relación entre la sociedad y la naturaleza, donde esta última sea considerada como la restricción fundamental del sistema de organización social imperante.

A partir de la constatación de la destrucción autoinducida del capital sobre la base de recursos naturales, se ha postulado la "segunda contradicción del capital, no explicita en El capital" (Leff 2003: 341), que se trata de la autoproducción de escasez de recursos, generada por el incremento del consumo productivo de recursos no renovables. 
Bibliografía

Altvater, Elmar y Birgit Mahnkopf. 2002. Las limitaciones de la g/obalización. Economía, ecología y política de la globalización. Siglo XXI Editores/UNAM, México.
Leff Enrique, Ecología y capital. Racionalidad ambiental, democracia participativa y desarrollo sustentable. México, Siglo XXI.

Marx, Karl. El capital, tomo I, sección tercera, cap. V. Siglo XXI. México, 2005 